M. OKA

KODAI MATH. J.

6 (1983), 419-433

\title{
ON THE BOUNDARY OBSTRUCTIONS TO THE JACOBIAN PROBLEM
}

\author{
By MutsuO OKA
}

\section{§1. Introduction}

Let $K$ be an algebraically closed field of characteristic zero and let $f(X, Y)$ and $g(X, Y)$ be polynomials with $K$-coefficients which satisfy the Jacobian condition :

$$
J(f, g)=f_{X} g_{Y}-f_{Y} g_{X}=1
$$

where $f_{X}, f_{Y}$ etc. are respective partial derivatives. The so-called Jacobian conjecture is the following.

(J.C. I ) "If (1.1) is satisfied, $X$ and $Y$ are polynomials of $f$ and $g$ ".

Typical examples are given by elementary transformations which are defined by finite compositions of the following transformations.

(i) $(f, g)=\left(a X+b Y+e, c X+d Y+e^{\prime}\right)$ where $a d-b c \neq 0$ or

(ii) $(f, g)=(X, Y+h(X))$ where $h(X)$ is an arbitrary polynomial.

By the theorem of Jung $[\mathrm{J}]$, (J.C.I) is equivalent to

(J.C. II) "If (1.1) is satisfied, $(f, g)$ is an elementary transformation".

Let $m=$ degree $(g)$ and let $g_{m}$ be the $m$-th homogeneous part of $g$. Among the various results about (J.C. I), the following is due to Abyankar [Ab]:

$g_{m}=0$ has at most 2 points in $P^{1}(K)$ if $(1.1)$ is satisfied by $f$ and $g$.

It is easy to prove that (J.C. II) is equivalent to (J.C. III) (See $\S 4$.):

(J.C. III) "If $g_{m}=0$ consists of two points, there is no polynomial $f$ such that $J(f, g)=1 "$.

In this paper, we study the necessary conditions ("boundary obstruction") of the boundaries of the Newton polygon $N(g)$ for the existence of $f$ such that $J(f, g)=1$. Unfortunately there exist polynomials which have no obstructions on the boundary. Our main results are in $\$ 6$ (Theorem (6.3) etc.).

Received February 1, 1983 


\section{$\S 2$. Jacobian problem for weighted homogeneous rational functions I.}

Parts of the results of this and the next section are obtained by Abyankar $[\mathrm{Ab}]$ but our approach is made from a different point of view.

Definition (2.1). A polynomial $f(X, Y)$ is called a weighted homogeneous polynomial of type $(\mathrm{a}, \mathrm{b} ; \mathrm{d})$ if $f\left(t^{\alpha} X, t^{b} Y\right)=t^{d} f(X, Y)$ for any $t \in K$. Here $a$ and $b$ are integers such that (i) $a$ and $b$ are coprime if $a b \neq 0$ and (ii) $(a, b)=(1,0)$ or $(0,1)$ if $a b=0 . \quad(a, b)$ are called the weights and $d$ is called the degree of $f$ with respect to the weights $(a, b)$. We denote it by $d=\operatorname{deg}_{(a, b)} f$.

EXAMPLE $(2.2) \quad X^{2}(Y+1)$ is a weighted homogeneous polynomial of type $(1,0 ; 2) . \quad X^{2}(X Y+1)$ is of type $(1,-1 ; 2)$.

Definition (2.3). A rational function $F(X, Y)=f(X, Y) / g(X, Y)$ is called a weighted homogeneous rational function of type $(a, b ; d)$ if $f$ and $g$ have the same weights $(a, b)$ and $d=\operatorname{deg}_{(a, b)} f-\operatorname{deg}_{(a, b)} g$. From the equation $F\left(t^{a} X, t^{b} Y\right)$ $=t^{d} F(X, Y)$, we obtain the Euler equation:

$$
d F(X, Y)=a X F_{X}(X, Y)+b Y F_{Y}(X, Y) .
$$

Proposition (2.5). Let $F(X, Y) \neq 0$ be a werghted homogeneous rational functron of type $(a, b ; d)$. Then $F(X, Y)$ can be unqquely factored as $c X^{p} Y^{q} \prod_{\imath=1}^{m}\left(X^{b}+c_{\imath} Y^{a}\right)^{n_{\imath}}$ where $c, c_{1}, \cdots, c_{m}$ are non-zero and $c_{\imath} \neq c_{\jmath}$ for $i \neq j$.

Proof. We may prove the assertion for a weighted homogeneous polynomial $f$. If $a b=0$, the assertion is nothing but the unique factorization property of a polynomial of one variable. Assume that $a b \neq 0$. We can write $F(X, Y)=$ $X^{p} Y^{q} f_{1}\left(X^{b}, Y^{a}\right)$ for some homogeneous polynomial $f_{1}(X, Y)$. Thus the assertion is reduced to the homogeneous case which is well known.

Definition (2.6). For a given $F(X, Y)$ as above, we define $p=\operatorname{val}_{X} F$, $q=\operatorname{val}_{Y} F$ and $n_{\imath}=\operatorname{val}_{\sigma_{\imath}} F$ where $\sigma_{\imath}=X^{b}+c_{\imath} Y^{a}$ for $\imath=1, \cdots, m$.

Proposition (2.7). ((17.4), $[\mathrm{Ab}])$. Let $F(X, Y)$ and $G(X, Y)$ be non-zero werghted homogeneous rational functions with the same weights $(a, b)$. Let $d_{1}=$ $\operatorname{deg}_{(a, b)} F$ and $d_{2}=\operatorname{deg}_{(a, b)} G$. Assume that $J(F, G)=0$. Then there exists a constant $c$ such that $F^{d_{2}}=c G^{a_{1}}$.

Proof. From the assumption $F_{X} G_{Y}-F_{Y} G_{X}=0$, we get $d_{1} F G_{Y}-d_{2} F_{Y} G=$ $\left(a X F_{X}+b Y F_{Y}\right) G_{Y}-F_{Y}\left(a X G_{X}+b Y G_{Y}\right)=0$. Similarly we get $d_{2} F_{X} G-d_{1} F G_{X}=0$. Thus taking the differential of $F^{d_{2}} / G^{d_{1}}$, we get 


$$
d\left(F^{d_{2}} / G^{d_{*}}\right)=\left\{\frac{d_{2} F_{X}}{F}-\frac{d_{1} G_{X}}{G}\right\} \frac{F^{d_{2}}}{G^{d_{1}}} d X+\left\{\frac{d_{2} F_{Y}}{F}-\frac{d_{1} G_{Y}}{G}\right\} F_{G^{d_{1}}}^{{ }^{d_{2}}} d Y=0 .
$$

This completes the proof.

The following lemma plays a key role in the following sections.

LEMMA (2.8). Let $F(X, Y)$ be a werghted homogeneous rational function of type $(a, b ; d)$. Let $\sigma$ be one of the divisors $X, Y$ and $X^{b}+c Y^{a}$ for some $c \neq 0$. Assume that $d \neq 0$ and $\operatorname{val}_{\sigma} F=0$. Then we have $\operatorname{val}_{\sigma} J(\sigma, F)=0$.

Proof. First observe that $J(\sigma, F)$ is a weighted homogeneous rational function of type $\left(a, b ; d^{\prime}-a-b\right)$ where $d^{\prime}=d+\operatorname{deg}_{(a, b)} \sigma$. Let $F(X, Y)=X^{p} Y^{q} \prod_{i=1}^{m}$ $\left(X^{b}+c_{\imath} Y^{a}\right)^{n_{\imath}}$. By the assumption, $d=p a+q b+\sum_{\imath=1}^{m} n_{\imath} a b \neq 0$. We put $\sigma_{\imath}=X^{b}+c_{\imath} Y^{a}$ for brevity's sake.

Case 1. Suppose $\sigma=X$. Then $\operatorname{val}_{\sigma} F=0$ implies $p=0$. Let $F_{1}, F_{2}$ and $G$ be rational functions. The following property of the Jacobian is used throughout this paper.

$$
J\left(G, F_{1} F_{2}\right)=J\left(G, F_{1}\right) F_{2}+J\left(G, F_{2}\right) F_{1} .
$$

As $J(X, Y)=1$ and $J\left(X, \sigma_{\imath}\right)=c_{\imath} a Y^{a-1}$, we have

$$
J(X, F)=q Y^{q-1} \prod_{\imath=1}^{m} \sigma_{\imath}^{n_{\imath}}+\sum_{\imath=1}^{m} n_{i} c_{i} a Y^{q+a-1} \sigma_{\imath}^{n_{\imath}-1} \quad\left(\prod_{\jmath \neq \imath} \sigma_{\jmath}^{n \jmath}\right) .
$$

Substituting $X=0$, we have

$$
J(X, F)_{\mid X=0}=\alpha Y^{n a+q-1}
$$

where $n=\sum_{\imath=1}^{m} n_{\imath}$ and $\alpha=(q+n a) \prod_{\imath} c_{\imath}^{n_{\imath}}$. As $d=q b+n a b=(q+n a) b$ is not zero, $\alpha$ is not zero. Thus (2.8.2) implies $\operatorname{val}_{X} J(X, F)=0$. (If $b$ is a negative integer, the sustitution $X=0$ should be replaced by $X^{\prime}=0$ where $X^{\prime}=X^{-1}$.)

The case that $\sigma=Y$ is treated in the exact same way.

Case 2. Suppose that $\sigma=X^{b}+c Y^{a}$ where $c \neq 0, c \neq c_{\imath}$ for $\imath=1, \cdots, m$. As $J\left(\sigma, \sigma_{\imath}\right)=\left(c_{i}-c\right) a b X^{b-1} Y^{a-1}$, we have

$$
\begin{aligned}
J(\sigma, F)= & -p a c X^{p-1} Y^{q+a-1} \prod_{\imath=1}^{m} \sigma_{\imath}^{n_{\imath}}+q b X^{p+b-1} Y^{q-1} \prod_{\imath=1}^{m} \sigma_{\imath}^{n_{\imath}} \\
& +\sum_{\imath=1}^{m} n_{\imath}\left(c_{i}-c\right) a b X^{p+b-1} Y^{q+a-1} \sigma_{\imath}^{n_{\imath}-1} \prod_{\jmath \neq \imath} \sigma_{\jmath}^{n_{\jmath}} .
\end{aligned}
$$

Restricting $J(\sigma, F)$ to $X^{b}+c Y^{a}=0$, we obtain

$$
J(\sigma, F)_{\mid \sigma=0}=d \prod_{\imath=1}^{m}\left(c_{i}-c\right)^{n_{\imath}} X^{p+b-1} Y^{n a+q-1} \neq 0
$$

by the assumption. This implies $\operatorname{val}_{\sigma} J(\sigma, F)=0$, completing the proof. 
THEOREM (2.9). Let $h(X, Y)$ be a weighted homogeneous polynomial of type $(a, b ; d)$ and suppose that $a>0, b>0$. Then there exists a weighted homogeneous rational function $\varphi(X, Y)$ such that $J(\varphi, h)=1$ if and only if either

(i) $h=\sigma_{1}^{p} \sigma_{2}^{q}$ where $\sigma_{1}$ and $\sigma_{2}$ are linear forms and $p \geqq 0, q \geqq 0, p \neq q$ or

(ii) $h=c X^{p}\left(Y+c^{\prime} X^{b}\right)^{q}$ where $c, c^{\prime} \neq 0$ and $a=1$ and $p$ and $q$ are as in (i), or

(iii) $h=c Y^{p}\left(X+c^{\prime} Y^{a}\right)^{q}$ where $c, c^{\prime} \neq 0, b=1$ and $p$ and $q$ are as in (i). (Compare with (18.9)-(18.12), [Ab].)

Proof. Let $h=c \sigma_{1}^{\alpha_{1}} \cdots \sigma_{k}^{\alpha} k$ be the factorization of $h$ as in Proposition (2.5). We assume that $\sigma_{1}=X, \sigma_{2}=Y$ and $\sigma_{2}=X^{b}+c_{2} Y^{a}$ for $i \geqq 3$ and $\alpha_{1}$ and $\alpha_{2}$ are non-negative and $\alpha_{\imath}(i \geqq 3)$ is positive. Suppose that $\varphi$ is a weighted homogeneous rational function such that $J(\varphi, h)=1$.

ASSERTION 1. (i) $\operatorname{val}_{\sigma_{\imath}} \varphi \geqq 1-\alpha_{\imath}$ if $\alpha_{\imath} \neq 0$,

$$
\geqq 0 \quad \text { if } \quad \alpha_{2}=0 \text {, }
$$

(ii) $\operatorname{val}_{\sigma} \varphi \geqq 0$ for any $\sigma \neq \sigma_{1}, \cdots, \sigma_{k}$.

Proof. The second part of (i) and (ii) can be treated in the same way. Let $\sigma$ be a divisor such that $\operatorname{val}_{\sigma} h=0$. Let $\varphi=\sigma^{\alpha} \varphi_{1}$ and $\operatorname{val}_{\sigma} \varphi_{1}=0$. Suppose $\alpha=\operatorname{val}_{\sigma} \varphi<0$. Then we have

$$
1=J(\varphi, h)=\alpha \sigma^{\alpha-1} \varphi_{1} J(\sigma, h)+\sigma^{\alpha} J\left(\varphi_{1}, h\right) .
$$

$\operatorname{val}_{\sigma} J(\sigma, h)=0$ by Lemma $(2.8)$ and $\operatorname{val}_{\sigma} J\left(\varphi_{1}, h\right) \geqq 0$ because $a$ and $b$ are positive. Taking $\operatorname{val}_{\sigma}$ of $(*)$, we get a contradiction $0=\alpha-1<0$. Now take $\sigma_{\imath}$ such that $\alpha_{\imath}>0$ and let $\beta_{i}=\operatorname{val}_{\sigma_{i}} \varphi$ and $\phi=\varphi^{\alpha_{i}} h^{-\beta_{\imath}}$.

Case 1. Suppose that $\operatorname{deg}_{(a, b)} \phi=\alpha_{i} \operatorname{deg}_{(a, b)} \varphi-\beta_{i} \operatorname{deg}_{(a, b)} h=0$. By the assumption $J(\varphi, h)=1$, we have

$$
\operatorname{deg}_{(a, b)} \varphi+\operatorname{deg}_{(a, b)} h=a+b>0 .
$$

Therefore $\frac{\beta_{\imath}}{\alpha_{\imath}}=\operatorname{deg}_{(a, b)} \varphi / \operatorname{deg}_{(a, b)} h>-1$ and this implies $\beta_{\imath}>-\alpha_{\imath}$ which is the assertion.

Case 2. Suppose that $\operatorname{deg}_{(a, b)} \phi \neq 0$. Then using (2.8) and the equality

$$
J(\psi, h)=\alpha_{i} \sigma_{\imath}^{\alpha_{i}-1} J\left(\psi, \sigma_{\imath}\right) \prod_{\jmath \neq \imath} \sigma_{\jmath}^{\alpha_{\jmath}}+\sigma_{\imath}^{\alpha_{i}} J\left(\psi, \prod_{\jmath \neq \imath} \sigma_{\jmath}^{\alpha_{j}}\right),
$$

we get $\operatorname{val}_{\sigma_{\imath}} J(\psi, h)=\alpha_{i}-1$. On the other hand, we can write $J(\phi, h)=J\left(\varphi^{\alpha}, h\right)$ $h^{-\beta_{\imath}}=\alpha_{i} \varphi^{\alpha_{i}-1} h^{-\beta_{\imath}}$. Therefore $\operatorname{val}_{\sigma_{\imath}} J(\psi, h)=\left(\alpha_{i}-1\right) \beta_{i}-\beta_{i} \alpha_{\imath}=-\beta_{\imath}$. Combining the two equalities, we get $\beta_{\imath}=1-\alpha_{\imath}$. This completes the proof of Assertion 1.

By Assertion 1 and the equality $a+b=\operatorname{deg}_{(a, b)} \varphi+\operatorname{deg}_{(a, b)} h$, we get the following inequality.

$$
\begin{gathered}
a+b \geqq\left(\alpha_{1}+\beta_{1}\right) a+\left(\alpha_{2}+\beta_{2}\right) b+\sum_{i \geqq 3}\left(\alpha_{i}+\beta_{\imath}\right) a b \\
\geqq\left(\alpha_{1}+\beta_{1}\right) a+\left(\alpha_{2}+\beta_{2}\right) b+(k-2) a b .
\end{gathered}
$$


Note that $\alpha_{i}+\beta_{\imath} \geqq 1$ if $\alpha_{\imath} \neq 0$ and $\alpha_{\imath}+\beta_{\imath} \geqq 0$ if $\alpha_{\imath}=0$.

First case: $k=2$. Then $h=c X^{\alpha_{1}} Y^{\alpha_{2}}$. Suppose that $\alpha_{1} \neq \alpha_{2}$. Then $\varphi=$ $\frac{1}{c\left(\alpha_{2}-\alpha_{1}\right)} X^{1-\alpha_{1}} Y^{1-\alpha_{2}}$ clearly satisfies $J(\varphi, h)=1$. Suppose that $\alpha_{1}=\alpha_{2}>0$. Then by the above inequalities, $\varphi$ must be written as $c^{\prime} X^{1-\alpha_{1}} Y^{1-\alpha_{2}}$ for some $c^{\prime}$. However this is absurd because $J(\varphi, h)=0$.

Second case: $k=3$. As $0<a+b-a b=-(a-1)(b-1)+1$, we must have $a$ $=1$ or $b=1$. ( $a=b=2$ is not allowed. ) Assume that $b=1$. As $a+1 \geqq\left(\alpha_{1}+\beta_{1}\right) a+$ $a+\left(\alpha_{2}+\beta_{2}\right)$, we must have $\alpha_{1}=0$ or $\alpha_{2}=0$ and $a=1$. (i) Suppose that $\alpha_{1}=0$. Then $h=c Y^{\alpha_{2}}\left(X+c_{3} Y^{a}\right)^{\alpha_{3}}$. If $\alpha_{2} \neq \alpha_{3}, \frac{1}{\left(\alpha_{2}-\alpha_{3}\right) c} Y^{1-\alpha_{2}}\left(X+c_{3} Y^{a}\right)^{1-\alpha_{3}}$ is the desired solution. If $\alpha_{2}=\alpha_{3}>0$, by the inequality (2.9.1), $\varphi$ must be $c^{\prime} Y^{1-\alpha_{2}}$ $\left(X+c_{3} Y^{a}\right)^{1-\alpha_{3}}$ which gives the contradiction $J(\varphi, h)=0$. (ii) Suppose that $\alpha_{2}$ $=0$ and $a=1$. By the same discussion as in the case $k=2, \alpha_{1} \neq \alpha_{3}$ is the necessary and sufficient condition for the existence of $\varphi$.

The case that $k=3$ and $a=1$ can be discussed in a similar way so that $h$ is either $c X^{\alpha_{1}}\left(X^{b}+c_{3} Y\right)^{\alpha_{3}}\left(\alpha_{1} \neq \alpha_{3}\right)$ or $c Y^{\alpha_{2}}\left(X+c_{3} Y\right)^{\alpha_{3}}\left(\alpha_{2} \neq \alpha_{3}\right)$.

Third case: $k=4$. As $a+b \geqq 2 a b$, we get $a=b=1$. By (2.9.1), $h$ must be $c \sigma_{3}^{\alpha_{3}} \sigma_{4}^{\alpha_{4}}$. As $\sigma_{3}$ and $\sigma_{4}$ are linear forms, we obtain, by the same discussion as in case 1 , that $\sigma_{3} \neq \sigma_{4}$ is the necessary and sufficient condition. The case that $k>4$ is clearly impossible by (2.9.1). This completes the proof of Theorem (2.9).

\section{$\S 3$. Newton polygon and the Jacobian problem.}

Let $f(X, Y)=\sum a_{\nu, \mu} X^{\nu} Y^{\mu}$ be a polynomial. We define the Newton polygon $N(f)$ by the convex hull of points $(\nu, \mu)$ for which $a_{\nu, \mu} \neq 0$. This is a compact polyhedron in $R^{2}$. For a face $\Delta$ ( $\Delta$ may be a vertex) of the boundary $\partial N(f)$, let $f_{\Delta}(X, Y)$ be the partial sum $\sum_{(\nu, \mu) \in \Delta} a_{\nu, \mu} X^{\nu} Y^{\mu}$. There are integers $a, b$ and $d$ such that $a$ and $b$ are coprime and $f_{\Delta}(X, Y)$ is a weighted homogeneous polynomial of type $(a, b ; d)$. If $\operatorname{dim} \Delta=1$ and $\Delta$ and the origin are not colinear, $a, b$ and $d$ are unique if we assume $d>0$.

Definition (3.1). We call $(a, b)$ the weights of $\Delta$. Let $\Gamma_{\infty}(f)$ be the union of $A$ 's which have positive weights. See Figure A.

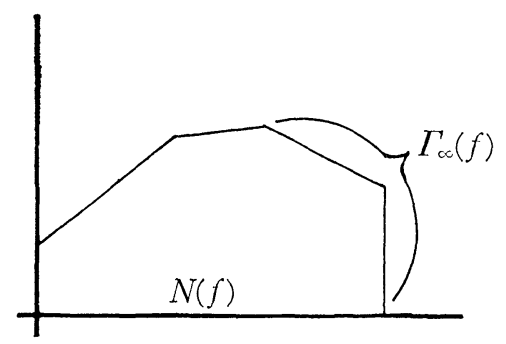

Figure A. 
Let $f(X, Y)$ and $g(X, Y)$ be polynomials which satisfy the Jacobian condition (1.1). Let $a$ and $b$ be coprime integers. Let $f=f_{-n^{\prime}}+f_{-n^{\prime}+1}+\cdots+f_{n}$ and $g=$ $g_{-m^{\prime}}+g_{-m^{\prime}+1}+\cdots+g_{m}$ be the gradations of $f$ and $g$ respectively. Namely $f_{i}(X, Y)$ $\sum_{a \nu+b \mu=\imath} a_{\nu \mu} X^{\nu} Y^{\mu}$. Note that we can write $f_{n}$ as $f_{\Delta}$ for some $\Delta \in \partial N(f)$.

We consider the equation $J(f, g)=1$. As $J\left(f_{\imath}, g_{j}\right)$ is a weighted homogeneous polynomial of degree $i+j-a-b, J(f, g)$ has the gradation $J(f, g)_{k}=\sum_{\imath+\jmath=k+a+b}$ $J\left(f_{\imath}, g_{j}\right)$. In particular, we have

PROPOSITION (3.2). $J\left(f_{n}, g_{m}\right)=0$ if $n+m \neq a+b$.

Write $g_{m}=h^{e}$ so that $e$ is a positive integer and $h$ is a square-free weighted homogeneous polynomial of degree $r$. $(e r=m)$.

PROPOSITION (3.3). For any $N>0$, there exists a rational function $\hat{g}(X, Y)$ such that $\hat{g}$ is a finite sum of werghted homogeneous rational functions so that $\operatorname{deg}_{(a, b)}\left(g-\hat{g}^{e}\right)<-N$.

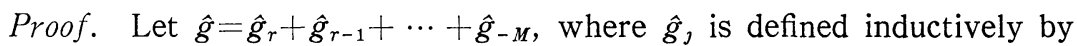

$$
\hat{g}_{r}=h \quad \text { and } \quad \sum_{\imath_{1}+\cdots+\imath_{e}=j} \hat{g}_{\imath_{1}} \hat{g}_{\imath_{2}} \cdots \hat{g}_{\imath_{e}}=g \text {, for } \jmath<m \text {. }
$$

For example, $\hat{g}_{r-1}=g_{m-1} / e h^{e-1}, \hat{g}_{r-2}=\left\{g_{m-2}-\left(\begin{array}{l}e \\ 2\end{array}\right) h^{e-2} \hat{g}_{r-1}^{2}\right\} / e h^{e-1}$. By definition, $\left(\hat{g}^{e}\right)_{j}=g$, for $j \geqq(e-1) r-M$. Thus the assertion is immediate if we take $M>0$ large enough.

LEMMA (3.4). If $m>0$, there exists a weighted homogeneous rational function $\varphi$ of degree $(a+b-m)$ such that $J\left(\varphi, g_{m}\right)=1$.

Proof. Take $N>0$ large enough and let $\hat{g}$ be as in Proposition (3.3). By Proposition (3.2) and Proposition (2.7), we can write $f_{n}=c_{q} h^{q}$ for some $c_{q} \neq 0$. $(q r=n)$. Then $\operatorname{deg}_{(a, b)}\left(f-c_{q} \hat{g}^{q}\right)<n$ and we have

$$
J\left(f-c_{q} \hat{g}^{q}, g\right)_{i}=J(f, g)_{i} \quad \text { for } \quad i \geqq 0 .
$$

To see this, let $R=g-\hat{g}^{e}$. Then $\operatorname{deg}_{(a, b)} R<-N$ and we have

$$
\begin{aligned}
J\left(\hat{g}^{q}, g\right)_{\imath} & =J\left(\hat{g}^{q}, \hat{g}^{e}+R\right)_{i} \\
& =J\left(\hat{g}^{q}, R\right)_{i}=0 \quad \text { for } \quad i \geqq 0,
\end{aligned}
$$

because $q r+\operatorname{deg}_{(a, b)} R-(a+b)<0$. Let $s$ be the minimal integer such that $s r>$ $a+b-m$. We repeat the same argument for $f-c_{q} \hat{g}^{q}$ and $g$ using (3.4.1).

I. Assume that $s \geqq 0$. By the inductive argument, we find constants $c_{q}$, $c_{q-1}, \cdots, c_{s}$ so that 


$$
\operatorname{deg}_{(a: b)}\left(f-\sum_{j=s}^{q} c_{j} \hat{g}^{\jmath}\right)<s r \quad \text { and } \quad J\left(f-\sum_{j=s}^{q} c_{j} \hat{g}^{\jmath}, g\right)_{i}=J(f, g)_{i}
$$

for $i \geqq 0$. Let $\varphi$ be the maximal gradation part of $f-\sum_{j=s}^{q} c_{j} \hat{g}^{\jmath}$. By (3.4.2), we have $\operatorname{deg}_{(a, b)} \varphi=a+b-m$ and $J\left(\varphi, g_{m}\right)=J(f, g)_{0}=1$.

II. Assume that $s<0$. We can find constants $c_{q}, \cdots, c_{0}$ so that

$$
\operatorname{deg}_{(a, b)}\left(f-\sum_{j=0}^{q} c_{j} \hat{g}^{\jmath}\right)<0 \text { and } J\left(f-\sum_{\jmath=0}^{q} c_{\jmath} \hat{g}^{\jmath}, g\right)_{\imath}=J(f, g)_{\imath}
$$

for $i \geqq 0$. Let $\breve{g}$ be the sum of weighted homogeneous factors of degree greater than $-N$ in the formal sum

$$
\begin{aligned}
\hat{g}^{-1} & =\hat{g}_{r}^{-1}\left(1+\hat{g}_{r-1} / \hat{g}_{r}+\cdots+\hat{g}_{-M} / \hat{g}_{r}\right)^{-1} \\
& =h^{-1} \sum_{j=0}^{\infty}(-1)^{\jmath} k^{\jmath} \quad \text { where } \quad k=\sum_{j=-M}^{r-1} \hat{g}_{J} / \hat{g}_{r} .
\end{aligned}
$$

We can write $\hat{g} \check{g}=1+S$ and $\operatorname{deg}_{(a, b)} S \leqq r-N$. Now we consider

$$
J\left(\check{g}^{\imath}, g\right)=J\left(\check{g}^{\imath}, \hat{g}^{e}+R\right) .
$$

It is easy to see that $\operatorname{deg}_{(a, b)} J\left(\check{g}^{2}, R\right)<0$ for $i \geqq 0$. We consider

$$
J\left(\check{g}^{\imath}, \hat{g}^{e}\right) \hat{g}^{\imath}=J\left(\check{g}^{\imath} \hat{g}^{2}, \hat{g}^{e}\right)=J\left((1+S)^{2}, \hat{g}^{e}\right)=J\left((1+S)^{2}-1, \hat{g}^{e}\right) .
$$

If $0<\imath \leqq-s$ and $M$ and $N$ are large enough, we see that $\operatorname{deg}_{(a, b)} J\left(\check{g}^{\imath}, \hat{g}^{e}\right)$ is negative. Thus we have

$$
\operatorname{deg}_{(a, b)} J\left(\check{g}^{\imath}, g\right)<0 \quad \text { for } \quad 0<\imath \leqq-s .
$$

The rest of the argument is exactly parallel to that of $I$. Suppose that we have chosen constants $c_{q}, \cdots, c_{\imath}$ such that $0 \geqq i>s$ and $\operatorname{deg}_{(a, b)} f^{(\imath)}<\imath r$ and $J\left(f^{(i)}, g\right)_{k}=J(f, g)_{k}$ for $k \geqq 0$ where $f^{(i)}=f-\sum_{j=0}^{q} c_{j} \hat{g}^{\jmath}-\sum_{k=\imath}^{-1} c_{k} \check{g}^{-k}$. By Proposition (2.7), we can find a constant $c_{\imath-1}$ such that $\operatorname{deg}_{(a, b)}\left(f^{(i)}-c_{\imath-1} \check{g}^{-\imath+1}\right)<(\imath-1) r$. Let $f^{(i-1)}=f^{(i)}-c_{\imath-1} \check{g}^{-\imath+1}$. Then by (3.4.5) we have

$$
J\left(f^{(i-1)}, g\right)_{k}=J(f, g)_{k} \quad \text { for } \quad k \geqq 0 .
$$

We stop the argument at $f^{(s)}$ and let $\varphi$ be $\left(f^{(s)}\right)_{a+b-m}$. Then $\varphi$ is the desired function.

COROLlary (3.5). Let $f$ and $g$ be as in Lemma (3.4). For any face or vertex $\Delta$ of $\Gamma_{\infty}(g), g_{\Delta}(X, Y)$ is one of (i ), (ii) and (iii) of Theorem (2.9).

Proof. Take positive integers $a, b$ and $d$ so that $g_{\Delta}(X, Y)$ is a weighted homogeneous polynomial of type $(a, b ; d)$. The assertion is immediate from 
Lemma (3.4) and Theorem (2.9). (Thus $\Gamma_{\infty}(g)$ has at most one 1-dimensional simplex.)

\section{§4. Equivalence of (J. C. II) and (J. C. III).}

We prove the equivalence of (J.C. II) and (J.C. III) in $\S 1$. Let $(f, g)$ be a polynomial pair which satisfies the Jacobian condition (1.1). Assume that $(f, g)$ is an elementary transformation. By an inductive argument on the number of compositions of transformations of type ( i ) and (ii) in $\S 1$ and by Propositions (2.7) and (3.2), we can see easily that $g_{m}=0$ has a unique solution in $P^{1}(K)$. Thus (J.C. II) implies (J.C. III). Now assume that (J.C. III) is verified. We prove (J.C. II) by the induction on $m=$ degree $g$.

Case 1. $\quad m=1$. Then we may assume that $g(X, Y)=X$. Then (1.1) can be solved directly so that we get $f(X, Y)=-Y+h(X)$ for some polynomial $h(X)$. This is clearly an elementary transformation.

Case 2. $m>1$. We may assume that $g_{m}(X, Y)=X^{m}$ by a linear change of coordinates if necessary. We consider the Newton polygon $N(g)$ and we take the face $\Delta$ of $\partial N(g)$ which has the point $(m, 0)$ at the end. By Corollary (3.5), we can write $g_{\Delta}(X, Y)$ as $c_{1} X^{p}\left(Y+c_{2} X^{b}\right)^{q}$ for some $b \geqq 2, p \geqq 0$ and $q>0$ where $c_{1}$ and $c_{2}$ are non-zero. We change the coordinates by $X^{\prime}=X$ and $Y^{\prime}=Y+c_{2} X^{b}$. Then it is easy to see that the degree of $g\left(X^{\prime}, Y^{\prime}\right)$ is strictly less than $m$. Thus $\left(f\left(X^{\prime}, Y^{\prime}\right), g\left(X^{\prime}, Y^{\prime}\right)\right)$ is an elementary transformation by the induction's hypothesis. Therefore $(f(X, Y), g(X, Y))$ is an elementary transformation.

\section{$\S 5$. Jacobian problem for weighted homogeneous rational functions II.}

Let $h(X, Y)$ be a weighted homogeneous polynomial of type $(a, b ; d)$ and we assume that $h$ is not a monomial and $a b \leqq 0$. In this section we study the necessary condition for the existence of a weighted homogeneous rational function $\varphi(X, Y)$ such that $J(\varphi, h)=1$. The Newton polygon $N(h)$ is a line segment $\overline{P Q}$ for some $P=(\alpha, \beta)$ and $Q=\left(\alpha^{\prime}, \beta^{\prime}\right)$ where $\alpha \leqq \alpha^{\prime}, \beta \leqq \beta^{\prime}$ and $\alpha+\beta<\alpha^{\prime}+\beta^{\prime}$.

Definition (5.1). We call $P$ and $Q$ the left and right end of $N(h)$ respectively.

( I ) Assume that $(a, b)=(1,-1)$. Then $N(h)$ is parallel to the line $Y-X$ $=0$. This case is exceptional by the following property. $\left({ }^{*}\right) \operatorname{deg}_{(1,-1)} J(F, G)=$ $\operatorname{deg}_{(1,-1)} F+\operatorname{deg}_{(1,-1)} G$ for any weighted homogeneous rational functions $F$ and $G$. Let $h(X, Y)=X^{\alpha} Y^{\beta} \prod_{\imath=1}^{k}\left(X Y+c_{\imath}\right)^{\nu}\left(c_{\imath} \neq 0\right)$. By the assumption, $k \geqq 1$ and $d=\alpha-\beta$. If $d$ is not zero, $\varphi=-X Y / d h$ is a desired function. Suppose that $d=0$. By the above property, $\varphi$ must be written as $(X Y)^{\gamma} \prod_{j=1}^{t}\left(X Y+d_{j}\right)^{\mu_{j}}$. However this gives a contradiction $J(\varphi, h)=0$. Thus we obtain 
THEOREM (5.2.). If $(a, b)=(1,-1)$, the necessary and sufficient condition for the existence of $\varphi$ is $d \neq 0$.

(II) Assume that $a+b \neq 0$ and $(a+b) d>0$. Changing the signs of $a, b$ and $d$ if necessary, we may assume that $a+b$ and $d$ are negative. We may also assume that $a \geqq 0>b$, taking the coordinates $X^{\prime}=Y$ and $Y^{\prime}=X$ if necessary. Let $h(X, Y)=X^{\alpha} Y^{\beta} \prod_{i=1}^{k}\left(X^{-b} Y^{a}+c_{\imath}\right)^{\nu_{i}}=X^{\tilde{\alpha}} Y^{\beta} \prod_{\imath=1}^{k}\left(Y^{a}+c_{\imath} X^{b}\right)^{\nu_{i}}$ where $\left\{c_{i}\right\}$ are non-zero and mutually distinct and $\tilde{\alpha}=\alpha-\Sigma \nu_{i} b$. Suppose that there exists a weighted homogeneous rational function $\varphi$ such that $J(\varphi, h)=1$. Let $\varphi(X, Y)=c_{0} X^{\gamma} Y^{\delta}$ $\prod_{j=1}^{k+t}\left(X^{-b} Y^{a}+c_{j}\right)^{\mu}$.

ASSERTION (5.3). (i) $\nu_{i}+\mu_{\imath} \geqq 1$ for $\imath \leqq k$ and (ii) $\mu$, $\imath$ non-negatıve for $j=k+1, \cdots, k+t$.

Proof. The proof is parallel to that of Assertion 1 in the proof of Theorem (2.9). First, the assertion (ii) is immediate from Lemma (2.8) and the following equality : $1=J(\varphi, h)=J\left(\varphi_{j}, h\right) \xi_{j}{ }^{\mu_{J}}+J\left(\xi_{\jmath}, h\right) \mu_{j} \xi_{j}{ }^{\mu_{j-1}} \varphi_{\text {, where }} \varphi_{\jmath}=\varphi / \xi_{j}{ }^{\mu_{\jmath}}$ and $\xi_{J}=Y^{a}$ $+c, X^{b}$. To prove (i), let $\phi_{i}=\varphi^{\nu_{i}} h^{-\mu_{2}}$. Assume that $\operatorname{deg}_{(a, b)} \psi_{i}=\nu_{i} \operatorname{deg}_{(a, b)} \varphi-$ $\mu_{i} d=0$. Combining this with the equality $\operatorname{deg}_{(a, b)} \varphi=-d+a+b$, we obtain

$$
-\mu_{\imath} / \nu_{i}=-\operatorname{deg}_{(a, b)} \varphi / d=1-(a+b) / d<1 .
$$

Thus we get $-\mu_{i}<\nu_{i}$ i.e. $\nu_{\imath}+\mu_{i} \geqq 1$. Assume that $\operatorname{deg}_{(a, b)} \psi_{i} \neq 0$. We consider two expressions of $J\left(\psi_{i}, h\right)$. First it is equal to $\nu_{i} \varphi^{\nu_{i}-1} h^{-\mu_{i}}$ as $J(\varphi, h)=1$ and $J(h, h)=0$. Secondly we can write $J\left(\phi_{i}, h\right)$ as $J\left(\phi_{i}, h / \xi_{i}^{\nu}\right) \xi_{i}^{\nu_{i}}+J\left(\phi_{i}, \xi_{i}\right) \nu_{i} \xi_{i}^{\nu_{i}-1} h / \xi_{i}^{\nu i}$. Comparing the $\operatorname{val}_{\xi_{i}}$ 's of both expressions, we get by Lemma (2.8)

$$
\left(\nu_{i}-1\right) \mu_{i}-\mu_{i} \nu_{i}=\nu_{i}-1 \text {. }
$$

Namely we get $\nu_{i}+\mu_{2}=1$, completing the proof of the assertion.

ASSERTION (5.4). Let $Q=\left(\alpha^{\prime}, \beta^{\prime}\right)$ be the right end of $N(h)$ and assume that $\alpha^{\prime}<\beta^{\prime}$. Then there exists a weighted homogeneous rational function $\varphi$ such that $J(\varphi, h)=1$ if and only if $a=\alpha=0$ and $k=1$.

Proof. Let $\phi=\varphi h$. Then by Assertion (5.3), $\phi$ is a Laurent polynomial (i. e. $\phi \in K\left[X, Y, X^{-1}, Y^{-1}\right]$ ) such that

$$
\phi=c_{0} X^{\alpha+\gamma} Y^{\beta+\delta} \prod_{\jmath=1}^{k+t}\left(X^{-b} Y^{a}+c_{\jmath}\right)^{\nu+\mu_{j}} \quad \text { and }
$$

$$
J(\phi, h)=h .
$$

Let $P^{\prime}=(\varepsilon, \delta)$ and $Q^{\prime}=\left(\varepsilon^{\prime}, \delta^{\prime}\right)$ be the left and right ends of $N(\phi)$ respectively. Let $L$ be the line which contains (1.1) and which is parallel to the segment $\overline{P Q}$. 
As $\operatorname{deg}_{(a, b)} \psi=a+b, L$ contains $P^{\prime}$ and $Q^{\prime}$. Let $P^{\prime \prime}$ and $Q^{\prime \prime}$ be the intersections of $L$ and $\overline{O P}$ and $\overline{O Q}$ respectively. See Figure $\mathrm{B}$.

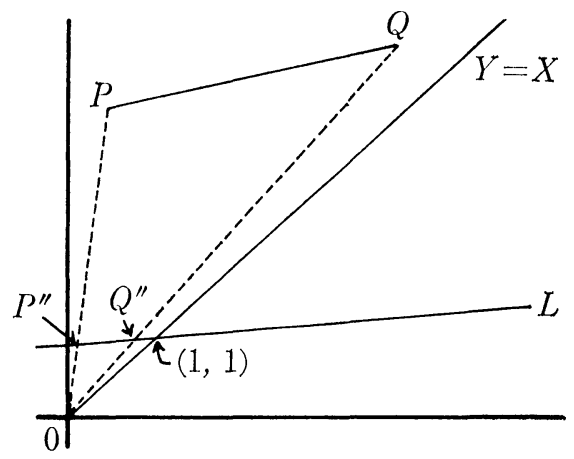

Figure B.

As $J\left(X^{\varepsilon^{\prime}} Y^{\delta^{\prime}}, X^{\alpha^{\prime}} Y^{\beta^{\prime}}\right)=\left(\varepsilon^{\prime} \beta^{\prime}-\delta^{\prime} \alpha^{\prime}\right) X^{\varepsilon^{\prime}+\alpha^{\prime}-1} Y^{\delta^{\prime}+\beta^{\prime}-1},\left(\varepsilon^{\prime}+\alpha^{\prime}-1, \delta^{\prime}+\beta^{\prime}-1\right)$ is the right end of $N(J(\phi, h))$ if $\varepsilon^{\prime} \beta^{\prime}-\alpha^{\prime} \delta^{\prime} \neq 0$. By (5.4.2), this implies that $Q^{\prime}=(1.1)$. Thus we get that $Q^{\prime}=(1.1)$ or $Q^{\prime}=Q^{\prime \prime}$ and $Q^{\prime \prime}$ is an integral point in the latter case. By the same discussion, $P^{\prime}=(1,1)$ or $P^{\prime \prime}$. In the latter case, $P^{\prime \prime}$ must be an integral point. In our case $Q^{\prime \prime}$ is not an integral point because $0<\alpha^{\prime}<\beta^{\prime}$. (See Figure B.) Thus $Q^{\prime}=(1,1) . \quad P^{\prime \prime}$ is an integral point if and only if $\overline{P Q}$ is parallel to the $X$-axis and $P$ is on the $Y$-axis. Namely $a=\alpha=0$. As $\psi$ is not a monomial by (5.4.1) and Assertion (5.3), the case that $P^{\prime}=Q^{\prime}=(1,1)$ is impossible. Thus $P^{\prime}=P^{\prime \prime}=(1,0)$ and $a=\alpha=0$. By (5.4.1), $k$ must be one. Thus $h=$ $Y^{\beta}\left(X+c_{1}\right)^{\nu_{1}}$ and $\varphi=c_{0} Y^{1-\beta}\left(X+c_{1}\right)^{1-\nu_{1}}$, where $c_{0}=1 /\left(\nu_{1}-\beta\right)$, is the desired solution.

Now we consider the case that $\alpha^{\prime} \geqq \beta^{\prime}$.

ASSERTION (5.5). $\alpha \neq \beta$ and $\alpha^{\prime} \neq \beta^{\prime}$.

Proof. Assume that $\alpha=\beta$. Then by the above discussion, $P^{\prime}=P^{\prime \prime}=(1,1)$. This is impossible because $J(\psi, h)$ cannot contain the non zero term $c X^{\alpha} Y^{\beta}$ as $J\left(X Y, X^{\alpha} Y^{\beta}\right)=0$. The case that $\alpha^{\prime}=\beta^{\prime}$ is impossible by the same argument.

We have two possible configurations.

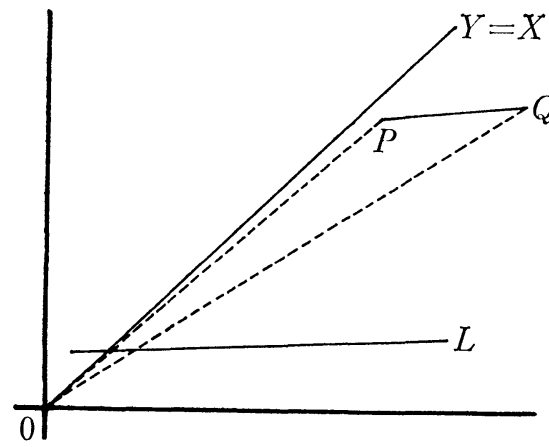

C. I.

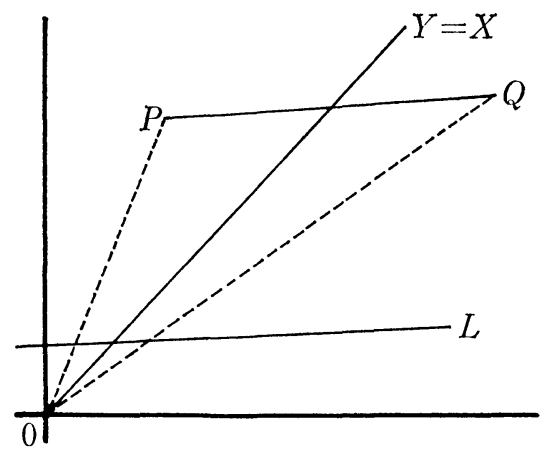

C. II. 
Case C.I. $\alpha>\beta$. As $P^{\prime \prime}$ is on the right side of $(1,1)$, we must have $P^{\prime}=$ $(1,1)$ to have a non-zero term $c X^{\alpha} Y^{\beta}$ in $J(\phi, h)$. If $Q^{\prime \prime}$ is not an integral point, we have that $Q^{\prime}=(1.1)$ which is impossible by (5.4.1). Thus it is necessary that $Q^{\prime \prime}$ is an integral point. (The sufficient condition is difficult to describe.)

Case C. II. First note that $P^{\prime \prime}$ is integral if and only if $a=\alpha=0$. Remember that $\psi$ is not a monomial. Thus if $(a, \alpha) \neq(0,0), Q^{\prime \prime}$ must be an integral point. We do not try to clarify the sufficient condition. (This is an algebraic condition on $\left\{c_{i}\right\}$ where $h(X, Y)=X^{\alpha} Y^{\beta} \prod_{\imath=1}^{k}\left(X^{b}+c_{\imath} Y^{a}\right)^{\nu_{\imath}}$ if $k, \alpha, \beta, \nu_{1}, \cdots, \nu_{k}$ are fixed.)

As a conclusion, we have:

TheOrem (5.5). Assume that $a \geqq 0>b, a+b<0$ and $d<0$. The following are necessary conditions for the existence of a werghted homogeneous rational function $\varphi$ such that $J(\varphi, h)=1$.

(i) The end points $P, Q$ of $N(h)$ are not on the line $Y-X=0$.

(ii) If $(a, \alpha) \neq(0,0), Q^{\prime \prime}$ must be an integral point. Namely there exists $a$ positıve integer $s$ such that $(1+s a) \alpha^{\prime}=(1-s b) \beta^{\prime}$. In partıcular, $Q$ must satısfy $\alpha^{\prime}>\beta^{\prime}$.

Remark (5.6). The above conditions are not sufficient for the existence of $\varphi$. We give some examples.

(A-I) Assume that $Q^{\prime \prime}=(1+a, 1-b)$ i.e. $(1+a) \alpha^{\prime}=(1-b) \beta^{\prime}$. By (5.4.1), we must have $k=1$ and $h(X, Y)=X^{\alpha} Y^{\beta}\left(X^{-b} Y^{a}+c_{1}\right)^{\nu_{1}}$. In this case we can solve $\phi$ as $c_{0} X Y\left(X^{-b} Y^{a}+c_{1}\right), c_{0}=1 /(\beta-\alpha) c_{1}$ and $\varphi=\psi / h$.

(A-II) Assume that $(1+2 a) \alpha^{\prime}=(1-2 b) \beta^{\prime}$ and $k=2$. By (5.4.1), $\psi$ must be $c_{0} X Y\left(X^{-b} Y^{a}+c_{1}\right)\left(X^{-b} Y^{a}+c_{2}\right)$. By an easy calculation, $\phi$ is a solution if and only if $c_{1}$ and $c_{2}$ satisfy the following equation:

$$
\left(\nu_{1} c_{1}+\nu_{2} c_{2}\right)\left|\begin{array}{c}
1-2 b, 1+2 a \\
\alpha^{\prime}+b, \beta^{\prime}-a
\end{array}\right|+\left(c_{1}+c_{2}\right)\left|\begin{array}{c}
1-b, 1+a \\
\alpha^{\prime}, \beta^{\prime}
\end{array}\right|=0 .
$$

(B) Assume that $(a+b) d<0$. We may assume that $a \geqq 0>b, a+b<0$ and $d>0$. This case is more difficult. The main reason is that Assertion (5.3) is not true in general. For example, let $h(X, Y)=X\left(X^{3} Y^{2}+c_{1}\right)^{2}$ and let $\varphi=\frac{-Y}{3}$ $\left(X^{3} Y^{2}+c_{1}\right)^{-3}\left(X^{3} Y^{2}+3 c_{1}\right)$. It is easy to see that $J(\varphi, h)=1$.

\section{§ 6. Boundary obstructions and further remarks.}

Let $(f, g)$ be a pair of polynomials which satisfy the Jacobian condition. We assume that $(f, g)$ is not an elementary transformation. Then by finite changes of coordinates of type (i) and (ii) in $\S 1$ if necessary, we may assume that $g_{m}(X, Y)=X^{p} Y^{q}(p>q>0$ and $m=p+q)$ where $m=$ degree $(g)$. Then the Newton polygon $N(g)$ is included in the rectangle $O P Q R$ in Figure $D$ by Corol- 
lary (3.5).

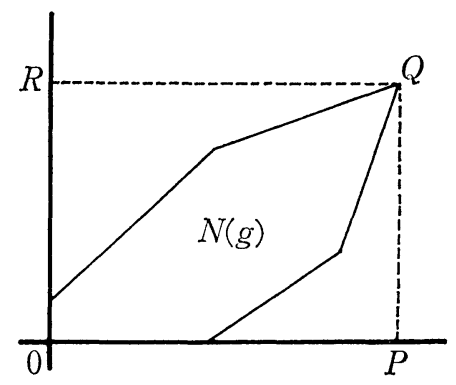

Figure D.

We may also assume that $0 \in N(g)$ and $0 \in N(f)$ by adding constants if necessary.

Lemma (6.1). Let $f$ and $g$ be as above. Then the polygons $N(f)$ and $N(g)$ are similar.

Proof. Let $\Delta$ be a 1-dimensional simplex of the boundary $N(g)$ which is not colinear with the origin. Let $(a, b ; d)$ be the weights of $\Delta$. Let $f_{\Delta^{\prime}}(X, Y)$ be the maximal gradation part of $f$ with respect to $(a, b)$ where $\Delta^{\prime}$ is a face of $N(f)$. Assume that $\operatorname{deg}_{(a, b)} f_{\Delta^{\prime}}+d=a+b$. Then we have $J\left(f_{\Delta^{\prime}}, g_{\Delta}\right)=1$. As $f_{\Delta^{\prime}}$ and $g_{\Delta}$ are polynomials, we may assume that, for example, $(1,0) \in \Delta$ and $(0,1)$ $\in \Delta^{\prime}$. Let $S$ and $S^{\prime}$ be the right ends of $\Delta$ and $\Delta^{\prime}$ respectively. See Figure E.

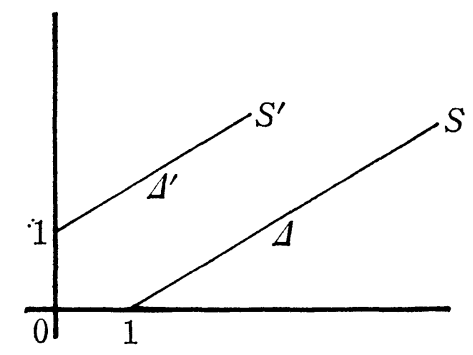

Figure E.

As $S$ and $S^{\prime}$ and the origin are not colinear, $J\left(f_{\Delta^{\prime}}, g_{\Delta}\right)$ contains a non-zero term $c X^{\alpha+\alpha^{\prime}-1} Y^{\beta+\beta^{\prime}-1}$ which is absurd. Thus we get $\operatorname{deg}_{(a, b)} f_{d^{\prime}}+d \neq a+b$. By (3.2), $f_{d^{\prime}}^{d^{\prime}} / g_{\Delta}^{d^{\prime}}$ is a constant where $d^{\prime}=\operatorname{deg}_{(a, b)} f_{\Delta^{\prime}}$. Let $S$ and $T$ (respectively $S^{\prime}$ and $T^{\prime}$ ) be the ends of $\Delta$ (respectively ends of $\Delta^{\prime}$ ). Then the triangle OST is similar to the triangle $O S^{\prime} T^{\prime}$ and $|\Delta| /\left|\Delta^{\prime}\right|=\overline{S T} / \overline{S^{\prime} T^{\prime}}=\overline{O S} / \overline{O S^{\prime}}=\overline{O T} / \overline{O T^{\prime}}$. As the faces $\Delta^{\prime} s$ of $N(g)$ as above are connected, the assertion is immediate.

COROLlary (6.2). Let $f$ and $g$ be as above. $N(f)$ and $N(g)$ contain the points $(1,0)$ and $(0,1)$. (Otherwise $J(f, g)$ cannot be 1.) 
Let $\Delta_{1}$ and $S_{2}$ be the two particular faces of $\partial N(g)$ which have $Q=(p, q)$ as the right ends. See Figure F.

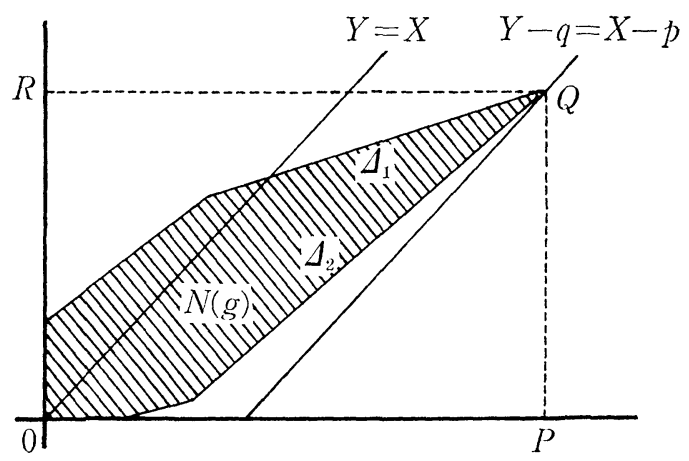

Figure F.

Assume that $\Delta_{1}=Q R$ and let $g_{\Delta_{1}}(X, Y)=Y^{q} \prod_{i=1}^{t}\left(X+c_{\imath}\right)^{\nu i}$. Then we take the new coordinates $X^{\prime}=X+c_{1}$ and $Y^{\prime}=Y$. Then $R$ is not contained in the Newton polygon of $g\left(X^{\prime}, Y^{\prime}\right)$. Note that the under part of $\partial N(g)$ i.e. $\{(x, y) \in \partial N(g)$; $p y \leqq q x\}$ remains unchanged. By the same device, we may assume that $P \notin \partial N(g)$. Now the results of $\S 5$ can be read as

THEOREM (6.5). (Boundary obstructions). For any simplex $\Delta$ of $\partial N(g)$ which is not colinear with the origin, there exists a werghted homogeneous rational functron $\varphi$ such that $J(\varphi, g)=1$. Let $\left(a_{\imath}, b_{i}\right)$ be the werghts of $\Delta_{\imath}(\imath=1,2)$. In part cular, we have the following.

(i) There exists a positive integer s such that $\left(1+s\left|a_{1}\right|\right) p=\left(1+s\left|b_{1}\right|\right) q$.

(ii) $a_{2}>0>b_{2}$ and $a_{2}+b_{2} \leqq 0$. (See Figure $F$.)

Remark (6.4). Let $(f, g)$ be a pair of polynomials which satisfy the Jacobian condition (1.1). Then $f$ and $g$ do not have any critical points in $K^{2}$ as functions from $K^{2}$ to $K$. However the converse is not true in general. For example, let $g_{1}(X, Y)=X+\sum_{i=1}^{k} c_{\imath}\left(X^{a} Y^{b}\right)^{2}$ and assume that $c_{k} \neq 0, a>1$ and $b>0$. It is easy to see that $g_{1}$ has no critical point. As $N\left(g_{1}\right)$ does not contain (0.1), there is no polynomial $f$ such that $J(f, g)=1$. A similar example is given by $g_{2}(X, Y)=$ $X+c_{2} X^{2}+\cdots+c_{m} X^{m}+a X^{n} Y$ where $n>m$ and $a \neq 0$.

Remark (6.5). Let $g(X, Y)$ be a polynomial with $g_{m}(X, Y)=X^{p} Y^{q}$ where $m=$ degree $(g)$ and $m=p+q$ and $p, q \geqq 1$. Let $\Delta$ be a 1 -dimensional simplex of $\partial N(g)$ such that $\Delta$ is not colinear with the origin. Write $g_{\Delta}(X, Y)=h_{\Delta}(X, Y)^{e(d)}$ where $h_{\Delta}(X, Y)$ is a weighted homogeneous, square free polynomial and $e(\Delta)$ is a positive integer. Let $e(g)$ be the greatest common divisor of such $e(\Delta)$ 's. The following is related to "Segre's Lemma" ([B-C-W]) and it might be well known 
to specialists.

Lemma (6.5.1). Assume that there exists a polynomial $f(X, Y)$ such that $J(f, g)=1$. Then $e(g)>1$.

Proof. For a 1-dimensional simplex $\Delta$ of $\partial N(g)$ which is not colinear with the origin, let $\Delta^{\prime}$ be the simplex of $\partial N(f)$ which corresponds to $\Delta$ by Lemma (6.1). Let $m=$ degree $(g)$ and $n=$ degree $(f)$. Assume that $e(g)=1$.

ASSERTION. $m$ divides $n$.

Proof. Let $\frac{n}{m}=\frac{n_{1}}{m_{1}}$ where $n_{1}$ and $m_{1}$ are coprime. Let $d(\Delta)$ be the degree of $h_{\Delta}(X, Y)$ with respect to the weights of $\Delta$ and let $d$ and $d^{\prime}$ be the respective degrees of $g_{\Delta}(X, Y)$ and $f_{\Delta^{\prime}}(X, Y)$. By the proposition (2.7) and (3.2), $f_{\Delta^{\prime}}(X, Y)$ $=c h_{\Delta}(X, Y)^{k(\Delta)}$ where $k(\Delta)$ is defined by $d^{\prime}=k(\Delta) d(\Delta)$. By Lemma (6.1), this implies that $d^{\prime}=d \frac{n_{1}}{m_{1}}=d(\Delta) e(\Delta) n_{1} / m_{1}$ is a multiple of $d(\Delta)$. As $e(g)=1$ by the assumption, this is possible only if $m_{1}=1$. This completes the proof of the assertion.

The rest of the argument is well known. Let $f_{1}(X, Y)=f(X, Y)-c g(X, Y)^{n_{1}}$. Then degree $\left(f_{1}\right)<$ degree $(f)$ and $J\left(f_{1}, g\right)=1$. By the inductive argument, we come to the situation that $J\left(f_{s}, g\right)=1$ and degree $\left(f_{s}\right)<$ degree $(g)$ which is impossible.

Remark (6.6). The final remark is a bit unfortunate for us: There exist polynomials without any obstructions from the boundary.

EXAMPLE (6.6.1). Let $g(X, Y)=Y^{n}\left(X^{2} Y+1\right)^{3 n}+X^{2 n}(X Y+1)^{4 n}-X^{6 n} Y^{4 n}$. Then $g$ has no obstruction on the boundary, i. e. there exists a weighted homogeneous rational function $\varphi_{\Delta}$ such that $J\left(g_{\Delta}, \varphi_{\Delta}\right)=1$ for any simplex $\Delta$ of $\partial N(g)$ which is not colinear with the origin. However there does not exist any polynomial $f(X, Y)$ such that $J(g, f)=1$ because $g$ has many critical points. We finish this paper with the following question.

Question: Is there any polynomial $g(X, Y)$ such that (i ) $g_{m}(X, Y)=X^{p} Y^{q}$ where $m=$ degree $g$ and $p+q=m$ and $p, q>0$ and (ii) $g$ has no obstruction on the boundary and (iii) $g$ has no critical point?.

\section{REFERENCES}

[Ab] Abyankar, S. S., Expansion techniques in algebraic geometry, Tata Inst. Furdamental Research, Bombay, 1977.

[J] Jung, H. W.E., Uber ganze birationale Transformatione der Ebene, J. Reine Angew. Math. 184 (1942), 161-174.

[B-C-W] Bass, H., Cornell, E. H. and Wright, D., The Jacobian conjecture, Bull. American Math. Soc., Vol. 7, Number 2, (1982), 287-330. 
ON THE BOUNDARY OBSTRUCTIONS

Department of Mathematics

Faculty of Sciences

Tokyo Institute of Technology

OH-OKayama, Meguro-ku,

TOKYO 